\title{
LA RETROALIMENTACIÓN COMO PARTE DE LA ENSEÑANZA DEL DISEÑO GRÁFICO EN EL AULA
}

\author{
Altamirano-Galván, Sandra Guadalupe \\ LA RETROALIMENTACIÓN COMO PARTE DE LA ENSEÑANZA DEL DISEÑO GRÁFICO EN EL AULA \\ Revista Legado de Arquitectura y Diseño, vol. 2019, núm. 26, 2019 \\ Universidad Autónoma del Estado de México, México \\ Disponible en: http://www.redalyc.org/articulo.oa?id=477961406003
}

Esta obra está bajo una Licencia Creative Commons Atribución-NoComercial-SinDerivar 4.0 Internacional. 


\title{
LA RETROALIMENTACIÓN COMO PARTE DE LA ENSEÑANZA DEL DISEÑO GRÁFICO EN EL
} AULA

\author{
FEEDBACK AS PART OF THE TEACHING OF \\ GRAPHIC DESING IN THE CLASSROOM \\ Sandra Guadalupe Altamirano-Galván \\ sandra.altamiranoglv@uanl.edu.mx \\ Universidad Autónoma de Nuevo León, México
} Revista Legado de Arquitectura y Diseño,
vol. 2019, núm. 26, 2019

Universidad Autónoma del Estado de México, México

Recepción: 04 Enero 2019

Aprobación: 13 Abril 2019

Redalyc: http://www.redalyc.org/ articulo.oa?id=477961406003

\section{BY-NC-ND}

Resumen: La presente investigación se enmarca en el proceso de enseñanza-aprendizaje del diseño gráfico en un contexto de educación superior, específicamente en el área de la identidad corporativa, dentro de un enfoque teórico-práctico. Se presentan los resultados obtenidos a partir de la enseñanza de las identidades en un aula de $4^{\circ}$ semestre de la Licenciatura en Diseño Gráfico de una Facultad perteneciente a una Universidad Pública, ubicada en la Ciudad de Monterrey, Nuevo León, México, dicha enseñanza tiene un enfoque constructivista que está basado en la retroalimentación docentealumno y alumno-alumno. El tema es indispensable para adentrarnos en la importancia que tiene la retroalimentación en el aprendizaje del alumno y en la mejora de sus propuestas de solución en la creación de diversos proyectos de identidad, a partir del desarrollo del espíritu crítico y la educación visual específica en esta rama del diseño. Para llevar a cabo esta investigación se hizo una revisión bibliográfica sobre los temas de enseñanza y retroalimentación, también se aplicaron encuestas para conocer la postura de los estudiantes con respecto al tema y además se realizó una investigación-acción en el aula mediante la participación de alumnos y docente, a fin de determinar los beneficios que la retroalimentación aporta al aprendizaje del alumno y con ello elaborar una propuesta de enseñanza de las identidades.

Palabras clave: aprendizaje, diseño, enseñanza, gráfico, identidad corporativa, retroalimentación.

Abstract: This research is part of the teaching-learning process of graphic design in a context of higher education, specifically in the area of corporate identity, within a theoreticalpractical approach. The results obtained from the teaching of identities in a fourth semester of the Degree in Graphic Design of a Faculty that belongs to a Public University, located in the City of Monterrey, Nuevo León, Mexico, are presented with a focus Constructivist and mainly based on teacher-student and student-student feedback. The theme is essential to get into the importance of feedback in student learning and the improvement of their solutions in the creation of various identity projects, from the development of the critical spirit and specific visual education in this branch of design. In order to carry out this research, a bibliographic review was made on teaching and feedback topics, surveys were also applied to know the students' position regarding the topic and an action research was also carried out in the classroom through the participation of students and teacher, in order to determine the benefits that feedback brings to the student's learning and thus develop a proposal for teaching identities.

Keywords: learning, design, teaching, graphic, corporate identity, feedback. 


\section{INTRODUCCIÓN}

La enseñanza del diseño gráfico se ha enfrentado por años a un desafío metodológico y estratégico para lograr un verdadero aprendizaje en el estudiante y con ello, un buen desempeño en su profesión. Desde la enseñanza teórica hasta la aplicación práctica, así como la evaluación, son aspectos que han generado preocupación y han sido grandes temas de discusión entre los académicos del diseño. La identidad corporativa se encuentra inmersa en el mundo globalizado y es una raíz del diseño gráfico. La enseñanza de la identidad en las aulas es un aspecto fundamental en la licenciatura, por lo cual tanto la teoría como la práctica deben tomarse en cuenta dentro de las estrategias de enseñanzaaprendizaje.

\section{LA IDENTIDAD CORPORATIVA SE EHCUEHTRA IHMERSA EH EL MUHDO GOBALLZADO y es una raíz del diseño gráfico.}

\section{DESARROLLO}

Según el paradigma constructivista, el alumno adquiere un aprendizaje significativo a partir de la práctica y del saber hacer, a través de metodologías que generen las competencias necesarias para desarrollarse en cualquier situación, dentro del mismo paradigma se toma en cuenta la retroalimentación como base sustancial del aprendizaje, sin embargo, en el contexto educativo del diseño gráfico, se escucha constantemente el reclamo de los alumnos cuando mencionan que sus maestros no les aportan ningún comentario sobre sus proyectos, lo que les impide conocer sus debilidades o fortalezas, generando descontento con la práctica educativa. Por lo tanto, en esta investigación, se busca conocer ¿Cuáles son los beneficios que la retroalimentación aporta al aprendizaje del alumno en la clase de identidad de la licenciatura en Diseño Gráfico?, también se busca saber ¿Cuáles son las teorías, pensamientos y estudios relacionadas con este tema en la práctica educativa?, ¿Cuál es el progreso del alumno a partir de la retroalimentación recibida durante el proceso de enseñanza-aprendizaje? y ¿Cómo sería una propuesta de enseñanza de la identidad en la licenciatura en Diseño Gráfico? Por lo tanto, los objetivos de la investigación se enfocan en determinar los beneficios que dicha comunicación del docente al alumno con respecto a sus proyectos, aporta al aprendizaje del alumno en la clase de identidad de la licenciatura en Diseño Gráfico, investigar teorías, pensamientos y estudios relacionados con la retroalimentación en la práctica educativa, identificar el progreso del alumno a partir de los comentarios recibidos durante el proceso educativo y elaborar una propuesta de enseñanza de la identidad en la licenciatura en Diseño Gráfico. 


\section{METODOLOGÍA}

El presente estudio tiene un enfoque mixto unido a la investigaciónacción, se realizó un análisis documental sobre los temas de la enseñanza y la retroalimentación, también se aplicaron encuestas para conocer la postura de los estudiantes con respecto al tema, además se realizó una investigación -acción en el aula mediante la participación de alumnos y docentes, a fin de determinar los bene ficios que la retroalimentación aporta al aprendizaje del alumno y con ello elaborar una propuesta de enseñanza de las identidades.

Dicha actividad se realizó durante el semestre enero-junio 2018, la autora puso en práctica una investigación en su clase de identidades, impartida en el $4^{\circ}$ semestre de la Licenciatura en Diseño Gráfico de una Facultad que pertenece a una Universidad Pública, ubicada en la Ciudad de Monterrey, Nuevo León, México. El curso es teórico-práctico y se imparte semestralmente. La población del estudio fue de 15 alumnos, 12 mujeres y 3 hombres.

El estudio se hizo en conjunto con 4 alumnos de $9^{\circ}$ semestre, inscritos en Estancia de Investigación, quienes apoyaron en las actividades de observación, recolección de datos, análisis y resultados.

$\mathrm{Al}$ inicio del semestre se aplicó una encuesta de Escala Likert de 5 niveles en donde se podían elegir las opciones totalmente de acuerdo, de acuerdo, ni en acuerdo ni en desacuerdo, en desacuerdo y totalmente en desacuerdo. La encuesta estuvo conformada por 7 ítems para conocer la postura de los alumnos con respecto al tema.

\section{INVESTIGACIÓN-ACCIÓN}

Se plantea un cronograma en el cual se programan los meses y semanas que conforman el semestre, cada semana hay una clase presencial, la cual está estructurada en teoría-práctica, así también hay actividades en línea que el alumno realiza desde la plataforma, y las actividades extra-aula en las cuales desarrollan los proyectos de diseño de identidades.

El cronograma (tabla 1) presenta 17 semanas, en las cuales hay 10 sesiones dedicadas a la retroalimentación, sin dejar de lado la teoría y la práctica en la clase, que es parte fundamental para el aprendizaje. Desde el primer día de clase los alumnos saben que tendrán este tipo de sesiones, existe una explicación por parte de la docente en la cual les comunica que la dinámica de la clase será de esa manera, les comenta la importancia de la retroalimentación y les pide respeto entre todos, así como críticas constructivas, haciendo énfasis en que la finalidad es obtener un aprendizaje significativo en ellos. 


\section{DINÁMICA DE LAS SESIONES DE RETROALIMENTACIÓN}

Una semana previa a la sesión, la docente les comenta a los alumnos que la siguiente semana habrá dinámica de retroalimentación y para ello les pide llevar sus propuestas de identidad en un archivo digital para proyectar al frente del aula.

Ya en la sesión, cada alumno de manera individual expone sus propuestas y la docente pide la participación de los alumnos-espectadores, ellos empiezan a dar sus comentarios y posteriormente la docente expresa su opinión, pueden existir preguntas, así como respuestas, para que las observaciones sean más claras y que el alumno al frente pueda obtener la mayor información posible para la mejora de su proyecto de identidad. Cabe mencionar que siempre existen dos sesiones de retroalimentación previas a la entrega de los proyectos y una sesión posterior, una vez que se evalúan los proyectos.

Tabla 1. Cronograma de la clase de identidades.

\begin{tabular}{|c|c|c|c|}
\hline Mes & Semana & Actividad aula & Retroalimentación \\
\hline \multirow[t]{2}{*}{ Enero } & $1^{2}$ semana & Presentación Tareas & \\
\hline & $2^{a}$ semana & Teoria-docente, participación alumnos & \\
\hline \multirow[t]{4}{*}{ Febrero } & $3^{a}$ semana & Actividad práctica aula/bocetaje & $\begin{array}{l}1^{\circledR} \\
\text { retroalimentación }\end{array}$ \\
\hline & $4^{a}$ semana & Presentación propuestas & $\begin{array}{l}2^{\mathrm{a}} \\
\text { retroalimentación }\end{array}$ \\
\hline & $5^{a}$ semana & $\begin{array}{l}\text { Presentación proyectos, no hay } \\
\text { retroalimentación }\end{array}$ & \\
\hline & $6^{\text {a }}$ semana & $\begin{array}{l}\text { Evaluación proyectos } \\
\text { Teoria-docente/participación alumnos }\end{array}$ & $\begin{array}{l}3^{a} \\
\text { retroalimentación }\end{array}$ \\
\hline \multirow[t]{3}{*}{ Marzo } & $7^{a}$ semana & Presentación propuestas & $\begin{array}{l}4^{a} \\
\text { retroalimentación }\end{array}$ \\
\hline & $8^{a}$ semana & Presentación propuestas & $\begin{array}{l}5^{a} \\
\text { retroalimentación }\end{array}$ \\
\hline & $9^{\circledR}$ semana & $\begin{array}{l}\text { Presentación proyectos, no hay } \\
\text { retroalimentación } \\
\text { Teoria-docente/participación alumnos }\end{array}$ & \\
\hline \multirow[t]{3}{*}{ Abril } & $\begin{array}{l}10^{\mathrm{a}} \\
\text { Semana }\end{array}$ & $\begin{array}{l}\text { Evaluación proyectos Teoría-docente } \\
\text { participación alumnos }\end{array}$ & \begin{tabular}{|l|}
$6^{\mathrm{a}}$ \\
retroalimentación
\end{tabular} \\
\hline & $\begin{array}{l}11^{\mathrm{a}} \\
\text { Semana }\end{array}$ & Teoria- docente participación alumnos & \\
\hline & $\begin{array}{l}12^{\mathrm{a}} \\
\text { Semana }\end{array}$ & Presentación propuestas & $\begin{array}{l}7^{\mathrm{a}} \\
\text { retroalimentación }\end{array}$ \\
\hline \multirow[t]{4}{*}{ Mayo } & $\begin{array}{l}13^{\mathrm{a}} \\
\text { Semana }\end{array}$ & Teoria-docente participación alumnos & \\
\hline & $\begin{array}{l}14^{\mathrm{a}} \\
\text { semana }\end{array}$ & Presentación propuestas & $\begin{array}{l}8^{\mathrm{a}} \\
\text { retroalimentación }\end{array}$ \\
\hline & $\begin{array}{l}15^{\mathrm{a}} \\
\text { semana }\end{array}$ & Presentación propuestas & $\begin{array}{l}9^{\mathrm{a}} \\
\text { retroalimentación }\end{array}$ \\
\hline & $\begin{array}{l}16^{\mathrm{a}} \\
\text { Semana }\end{array}$ & Entrega proyectos finales & \\
\hline Junio & $\begin{array}{l}17^{\mathrm{a}} \\
\text { Semana }\end{array}$ & $\begin{array}{l}\text { Revisión proyectos } \\
\text { finales /evaluación-cierre. }\end{array}$ & $\begin{array}{l}10^{\mathrm{a}} \\
\text { retroalimentación }\end{array}$ \\
\hline
\end{tabular}

Fuente: Elaboración propia.

Para la recolección y análisis de los datos se realizó un instrumento en donde se fue reuniendo información en cada sesión de retroalimentación 
con respecto a la calidad de los proyectos presentada por los alumnos, y con esto, determinar el beneficio que el ejercicio aporta al aprendizaje del alumno, para ello se desarrolló una rúbrica que toma en cuenta los aspectos fundamentales en la creación de proyectos de identidad, que es la misma que utiliza la docente para evaluación y para retroalimentación hacia los alumnos.

Para determinar la calidad de los proyectos, con respecto a la rúbrica se establecieron tres niveles, los cuales son: Calidad Buena, Calidad Intermedia y Calidad Baja (tabla 2).

Tabla 2. Niveles de calidad de los proyectos.

\begin{tabular}{|l|l|}
\hline $\begin{array}{l}\text { NIVELES DE } \\
\text { CALIDAD DE } \\
\text { POS } \\
\text { PROYECTOS }\end{array}$ & DESCRIPCIÓN \\
\hline BUENA & $\begin{array}{l}\text { Resuelve de manera } \\
\text { adecuada el proyecto, con } \\
\text { respecto a los 9 conceptos de } \\
\text { la rúbrica. }\end{array}$ \\
\hline INTERMEDIA & $\begin{array}{l}\text { Resuelve de manera regular el } \\
\text { proyecto, con respecto a los 9 } \\
\text { Conceptos de la rúbrica. }\end{array}$ \\
\hline BAJA & $\begin{array}{l}\text { No resuelve de manera } \\
\text { adecuada el proyecto, con } \\
\text { respecto a los 9 conceptos de } \\
\text { la rúbrica. }\end{array}$ \\
\hline
\end{tabular}

Fuente: Elaboración propia.

Así también se realizó un instrumento en el cual se recolectó la información para conocer el nivel de participación de los alumnos en el ejercicio de retroalimentación, y con ello, determinar cómo el ejercicio le permite desarrollar el espíritu crítico con respecto al tema de las identidades. Para determinar la participación de los alumnos, se establecieron tres niveles, los cuales son: Participación Alta, Participación Media, Participación Baja (tabla 3).

Tabla 3. Niveles de participación en la retroalimentación.

\begin{tabular}{|l|l|}
\hline $\begin{array}{l}\text { NIVELES DE } \\
\text { PARTICIPACIÓN } \\
\text { DE LOS } \\
\text { ALUMNOS }\end{array}$ & DESCRIPCIÓN \\
\hline ALTA & $\begin{array}{l}\text { Participa de manera activa en } \\
\text { la retroalimentación y con } \\
\text { mayor en foque crítico. }\end{array}$ \\
\hline MEDIA & $\begin{array}{l}\text { Participa de manera regular } \\
\text { en la retroalimentación y con } \\
\text { menor enfoque crítico. }\end{array}$ \\
\hline BAJA & $\begin{array}{l}\text { No participa en la } \\
\text { retroalimentación. }\end{array}$ \\
\hline
\end{tabular}

Fuente: Elaboración propia. 


\section{RESULTADOS}

\section{Teorias, pensamientos y estudios relacionadas con la retroalimentación en la práctica educativa}

Con base en la información recopilada y analizada tenemos que la retroalimentación es "la acción valorativa del proceso formativo en un momento determinado del curso, es como la «fotografía» del estado de conocimiento adquirido por el estudiante" (Román, 2009: 14).

La retroalimentación es el conjunto de palabras que permitirán al alumno obtener información acerca de la valoración que otras personas, ya sea el docente o los compañeros, hacen acerca de sus proyectos.

"En términos educativos podemos definirla como aquella información que se utiliza para reducir la diferencia entre los resultados de aprendizaje obtenidos por el estudiante y los resultados de aprendizaje esperados" (García, 2015: 8). Es importante señalar lo mencionado en esta cita en cuanto a "reducir", es decir, la retroalimentación busca acortar la brecha del desconocimiento, al señalar los puntos débiles, para que sean mejorados por quien recibe dicha información.

Beltrán (2005: 489) menciona que la retroalimentación, en su contexto instruccional o situación de enseñanza-aprendizaje se da o se crea cuando el maestro quiere que el alumno conozca la calidad de sus respuestas con los aciertos y con los errores; este tipo de información debiera darse al menos en estas situaciones:

a) Ante respuestas rápidas y correctas hacérselo saber al alumno con brevedad y firmeza.

b) Ante respuestas correctas, pero dubitativas hacérselo saber dándole seguridad e información adicional.

c) Ante respuestas incorrectas poco importantes, hacérselo saber de forma rápida y breve.

d) Ante respuestas incorrectas relativas a informaciones importantes, hacérselo saber mediante información adicional, pistas o indicios que ayuden al alumno a encontrar la respuesta correcta.

Con esto, podemos darnos cuenta que la retroalimentación es importante en la mayoría de los casos o de las situaciones que surgen en el proceso de enseñanza-aprendizaje, tanto en las respuestas correctas como en las incorrectas, es decir, no solamente señalar los errores, sino también los aciertos, para así reafirmar la seguridad del alumno.

\section{LA RETROALIMENTACIÓN ES EL CONJUNTO DE PALABRAS \\ que permitión al alumno obtener informacón acenca de la valoración que otras personas, YASEAEL DOCENTE O LOS COMPAREROS. HACEN ACERCA DE SUS PROYECTOS.}

Gorriez (2017: 32) menciona que "hay que encontrar el clima apropiado para realizar el Feed Back (retroalimentación), es conveniente 
que sea un momento de tranquilidad, cuidando las formas y las palabras, y siempre explicitando a los alumnos que el llamado error forma parte del proceso de aprendizaje". Es importante que el docente genere el ambiente idóneo al iniciar esta retroalimentación para que el alumno se sienta tranquilo y confiado de que la actividad tiene fines positivos que le permitan un mayor aprendizaje.

Esto se reafirma en cuanto a que "la retroalimentación es fundamental para que los estudiantes tengan claro dónde se ubican en su proceso; se constituye en una excelente alternativa de aprendizaje, ya que así se fortalecen los aspectos positivos y se reconoce en situaciones difíciles como una oportunidad de mejoramiento" (Morales y Restrepo, 2015: 91).

Rodríguez y Gil (2011: 133) mencionan que las condiciones que Gibbs y Simpson (2004-2005) consideran como necesarias para que la retroalimentación favorezca el aprendizaje del estudiante son:

- Cantidad y tiempo de retroalimentación.

- Se ofrece la suficiente retroalimentación y con el nivel de detalle suficiente.

- La retroalimentación se ofrece lo suficientemente rápido para que resulte de utilidad al estudiante.

- Calidad de la retroalimentación.

- La retroalimentación se centra sobre el aprendizaje antes que sobre las calificaciones o sobre los propios estudiantes.

- La retroalimentación es comprensible para el estudiante.

- Respuesta del estudiante a la retroalimentación.

- La retroalimentación es bien recibida y atendida por el estudiante.

- La retroalimentación es puesta en práctica por el estudiante para mejorar su trabajo o su propio aprendizaje.

\section{EL EJERCICIO DE RETROALIMENTACIÓN DEBE DARSE DE FORMA ADECUADA, SUFICIENTE, PROPICIA Y BIEN EST RUCTURADA, DE MANERA QLE}

el estudiante lo pueda aceptar positivamente, pero además que lo haga propio.

El ejercicio de retroalimentación debe darse de forma adecuada, suficiente, propicia y bien estructurada, de manera que el estudiante lo pueda aceptar positivamente, pero además que lo haga propio y lo integre como parte de su conocimiento, "es conveniente centrarse en aspectos principales que el docente considera deben ser trabajados, mejorados o modificados. Para poder realizar la devolución adecuada, el docente debiera en primera instancia priorizar, jerarquizar y elegir los contenidos sobre los que centrará su devolución" (Gorriez, 2017: 32).

En lo que respecta a la enseñanza del diseño gráfico, Zatarain (2015) habla de la falta de bases pedagógicas en los actores del proceso, así como de las estrategias didácticas necesarias para la formación de los diseñadores gráficos de la actualidad. 
Es necesario utilizar el enfoque constructivista y a través del mismo, lograr una mejora con respecto a la relación enseñanza-aprendizaje entre el maestro y alumno, generando un ambiente propicio para la apropiación del conocimiento, todo esto basado en una línea de comunicación horizontal que da lugar al debate y a las aportaciones por todos los miembros que forman parte del grupo (Mendivil, Martínez y Camacho, 2017: 5).

Generar el ambiente propicio para la retroalimentación en donde, tanto el docente como los mismos alumnos comparten aportes entre sí, es fundamental para el aprendizaje y la mejora de la calidad de los proyectos de diseño gráfico, además permite el desarrollo del espíritu crítico y la competencia comunicativa.

También cabe mencionar que "la concepción sobre el diseño gráfico que tenga una institución y todos los involucrados, como docentes, estudiantes, egresados y medio laboral, será determinada por el entorno, la retroalimentación que los estudiantes reciban y por supuesto con la formación y experiencia de quienes enseñan y proporcionan esa mismo retroalimentación, los docentes" (Mendivil, Martínez y Camacho, 2017: 4).

La retroalimentación se enfoca desde dos vertientes, por un lado, la que ofrece el profesor orientando el diálogo; y por otro, la que fomenta la participación de los mensajes, a través de técnicas de motivación vinculadas al contenido (Torres, Inés y Valdiviezo, 2013: 7).

Es importante que los docentes puedan verificar que el estudiante haya comprendido aquello que se le solicita que modifique. En muchas oportunidades no comprenden adecuadamente lo que se les sugiere y debido a ello, vuelven a cometer el mismo error (Gorriez, 2017: 32).

El aprendizaje es más fácil cuando la retroalimentación es relevante, y mejora cuando se transita de un ambiente benévolo a uno exigente, el cual demanda precisión. Cuando la retroalimentación es deficiente, ambigua o con ruido y el ambiente es benévolo, el agente puede permanecer atrincherado en una concepción supersticiosa del mundo, sin consecuencia o costo alguno. $\mathrm{Y}$ no puede seguir atrincherado cuando la retroalimentación es deficiente y los errores producen fuertes consecuencias (Lara, 2011: 106).

\section{Progreso del alumno a partir de la retroalimentación recibida durante el proceso de enseñanza-aprendizaje}

\section{RESULTADOS ENCUESTA DE INICIO DE SEMESTRE}

Al inicio del semestre se aplicó una encuesta de Escala Likert de 5 niveles en donde se podían elegir las opciones totalmente de acuerdo, de acuerdo, ni en acuerdo ni en desacuerdo, en desacuerdo y totalmente en desacuerdo. La encuesta estuvo conformada por 7 ítems, para conocer la postura de los alumnos con respecto al tema.

Con respecto a esta encuesta, se obtuvieron resultados favorables de la postura de los alumnos en cuanto al tema estudiado, puesto que un $74 \%$ estuvo totalmente de acuerdo en que la retroalimentación docentealumno es importante, mientras que el $26 \%$ menciona estar de acuerdo 
con ello (figura 1). Así también, el 60\% de los encuestados estuvo totalmente de acuerdo con que la retroalimentación le ayuda a mejorar sus proyectos, mientras que el $27 \%$ se mostró de acuerdo, y el $13 \%$ no estuvo ni en acuerdo ni en desacuerdo (figura 2).

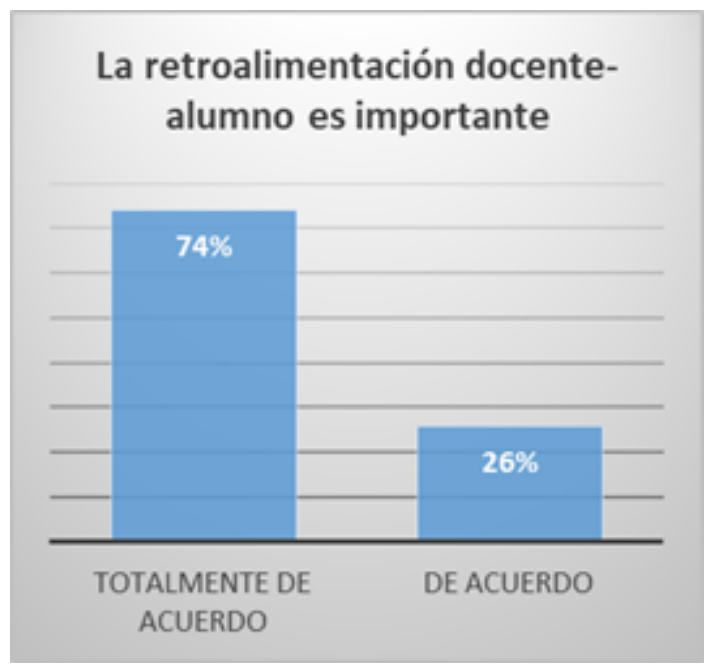

Figura 1. Resultados ítem 1.

Fuente: Elaboración propia.

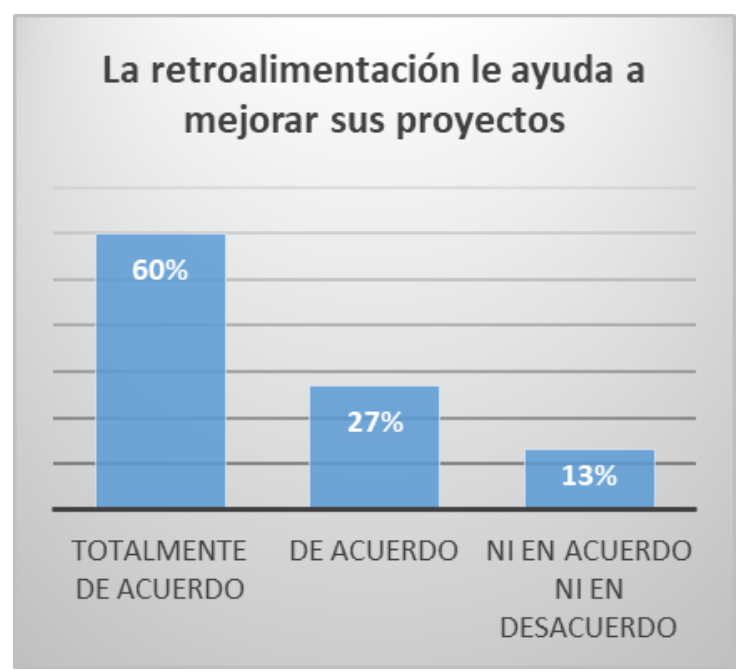

Figura 2. Resultados ítem 2.

Fuente: Elaboración propia.

Asimismo, un 60\% de los alumnos encuestados mencionó estar totalmente de acuerdo en que la retroalimentación le ayuda a aprender mejor, mientras que el $40 \%$ dijo estar de acuerdo (figura 3). Y en relación a esto, el $13 \%$ de los alumnos encuestados menciona estar totalmente de acuerdo en que cuando sus compañeros le dan retroalimentación de los proyectos, aprenden más, así también el $67 \%$ se encuentra de acuerdo, mientras que el $20 \%$ no estuvo ni en acuerdo ni en desacuerdo (figura 4). 


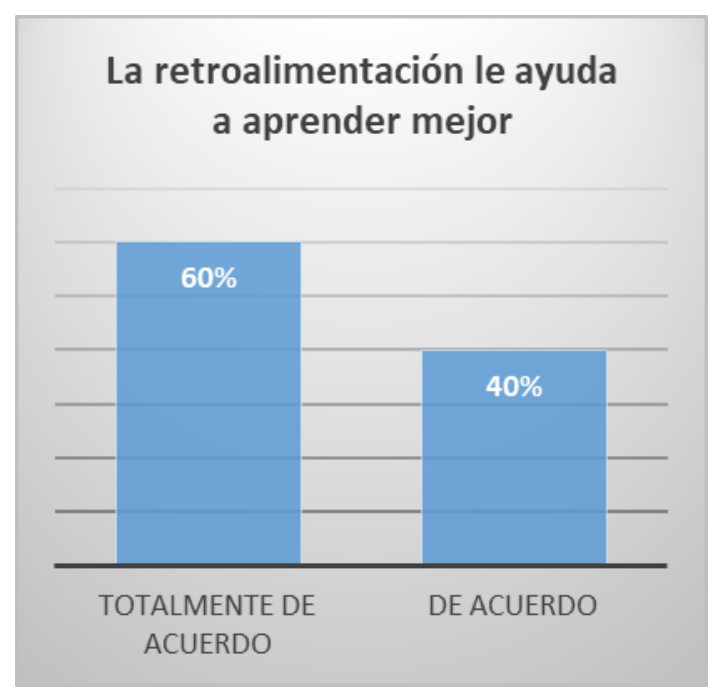

Figura 3. Resultados ítem 3.

Fuente: Elaboración propia.

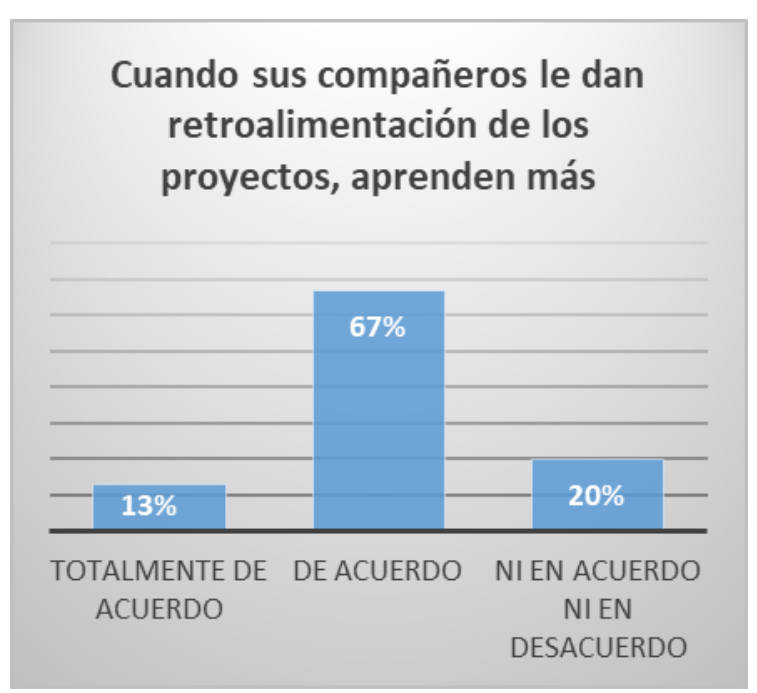

Figura 4. Resultados ítem 4.

Fuente: Elaboración propia.

El 13\% de los encuestados mencionó estar totalmente de acuerdo en que prefiere que los docentes no le den su opinión sobre sus proyectos, el $13 \%$ mencionó estar de acuerdo con esta afirmación, mientras que el 13\% no estuvo ni en acuerdo ni en desacuerdo, por otro lado, el 34\% dijo estar en desacuerdo y el $27 \%$ estuvo totalmente en desacuerdo (figura 5). 


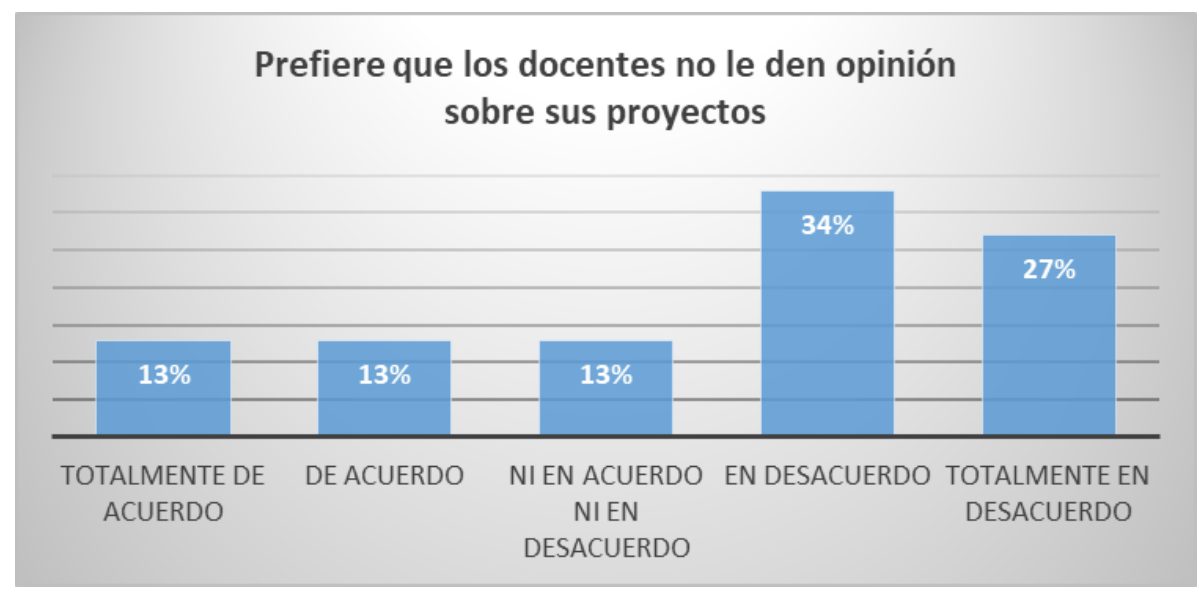

Figura 5. Resultados ítem 5.

Fuente: Elaboración propia.

En lo que respecta al área de la enseñanza de la identidad en diseño gráfico, el $80 \%$ de los encuestados mencionó estar totalmente de acuerdo en que considera importante la retroalimentación para su aprendizaje en el desarrollo de identidades, mientras que el 20\% dijo estar de acuerdo (figura 6).

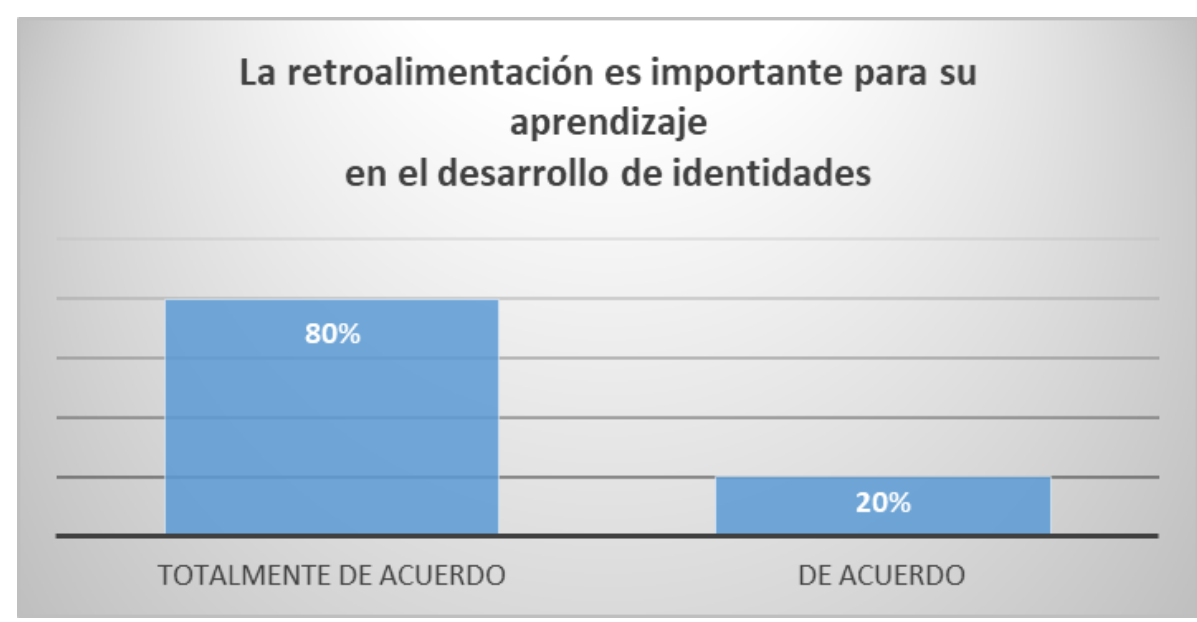

Figura 6. Resultados ítem 6.

Fuente: Elaboración propia.

Y por último, un 13\% estuvo totalmente de acuerdo en que durante su estancia en la Facultad, siempre ha recibido retroalimentación de sus proyectos por parte de los docentes, mientras que un $47 \%$ dijo estar de acuerdo, un $27 \%$ no estuvo ni en acuerdo ni en desacuerdo y un $13 \%$ se mostró en desacuerdo (figura 7). 


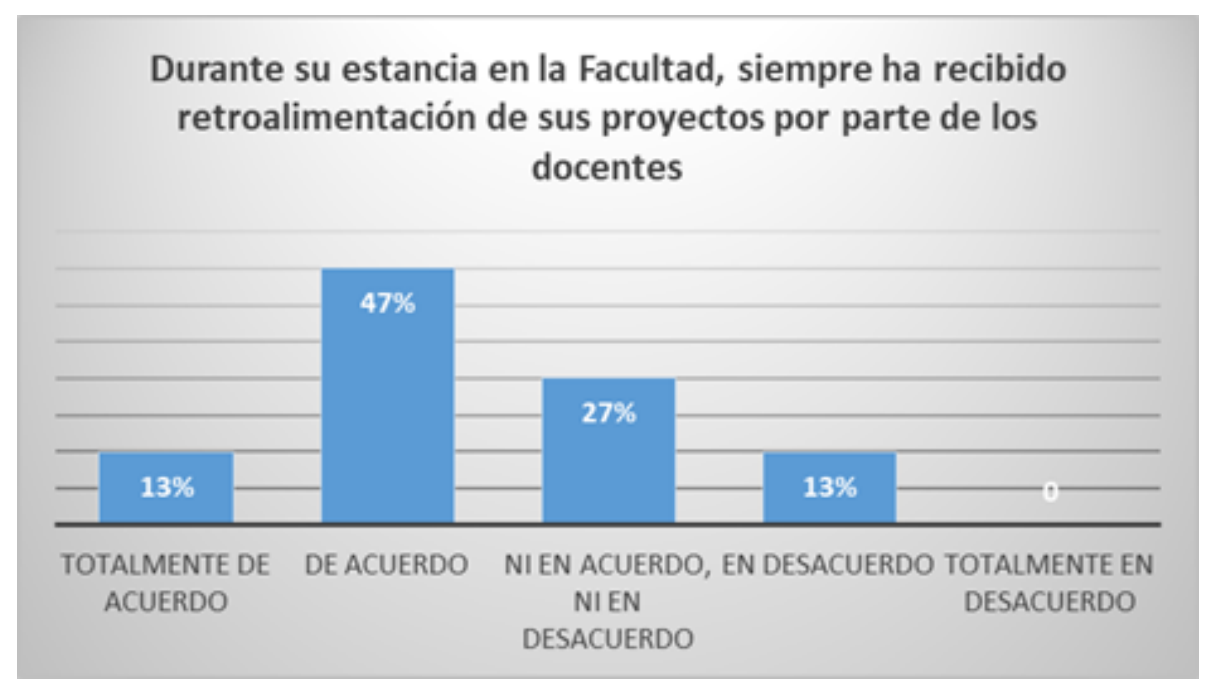

Figura 7. Resultados ítem 7.

Fuente: Elaboración propia.

\section{RESULTADOS INVESTIGACIÓN-ACCIÓN}

En cuanto a la información recolectada en cada sesión de retroalimentación con respecto a la calidad de los proyectos presentada por los alumnos, se encontró un avance significativo en ellos. En la figura 8 , se observa el avance de los alumnos desde el primer ejercicio de retroalimentación hasta el último, se muestra que en las primeras sesiones, existe una mayor cantidad de alumnos con calidad baja en el desarrollo de sus proyectos de identidad.

Así también se muestra una mayor cantidad de alumnos con una calidad intermedia en las primeras sesiones de retroalimentación, mientras que conforme va aumentando el número de sesiones de retroalimentación, también va elevándose el número de alumnos con una calidad buena en el desarrollo de sus proyectos, observando también una disminución del número de alumnos con calidad intermedia y calidad baja en sus proyectos. 


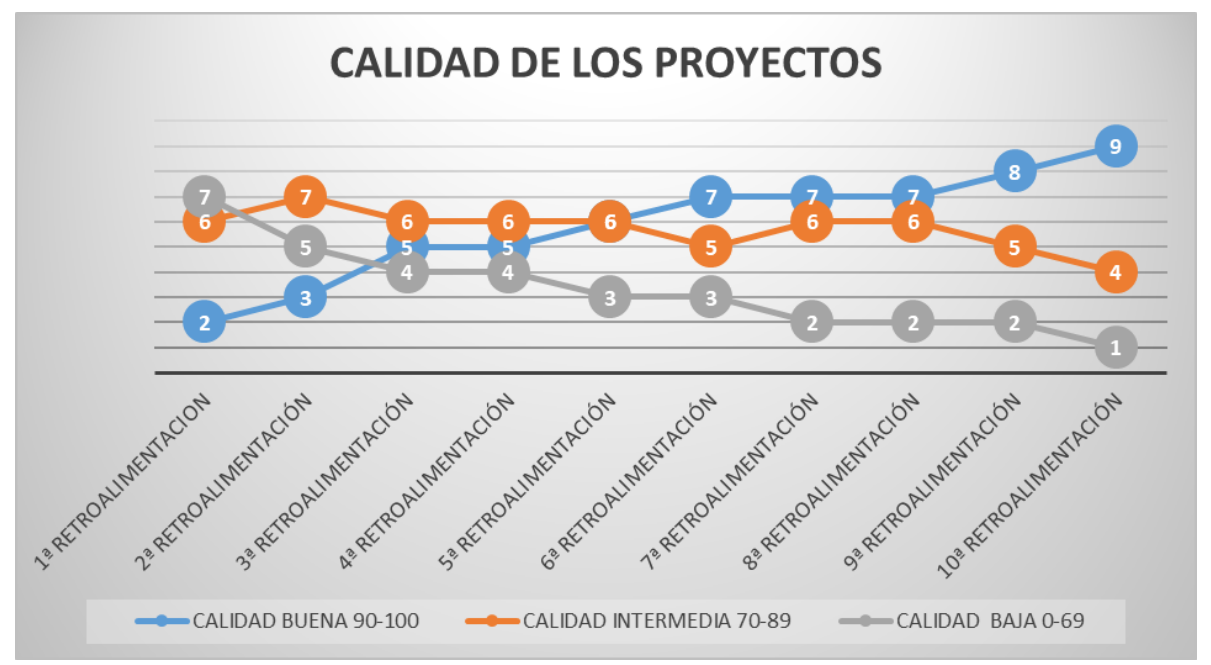

Figura 8. Calidad de los proyectos por sesión de retroalimentación.

Fuente: Elaboración propia.

En cuantoa la información recolectada en cada sesión de retroalimentación con respecto al nivel de participación de los alumnos en el ejercicio, en la figura 9 , se observa que en las primeras sesiones la participación era en su mayoría baja , tiene muy poca participación media, y nula participación alta

Conforme va avanzando el número de sesiones de retroalimentación, vemos que en la sexta sesión la participación va aumentando y encontramos niveles muy similares entre la participación alta, media y baja.

Y vemos un avance significativo mayor en las últimas sesiones, en donde la participación baja disminuye casi en su totalidad, mientras que la participación media y alta, aumentan, teniendo un número casi igual entre las dos.

Lo que permite observar que conforme las sesiones van avanzando, los alumnos van participando cada vez más en la retroalimentación alumnoalumno. 


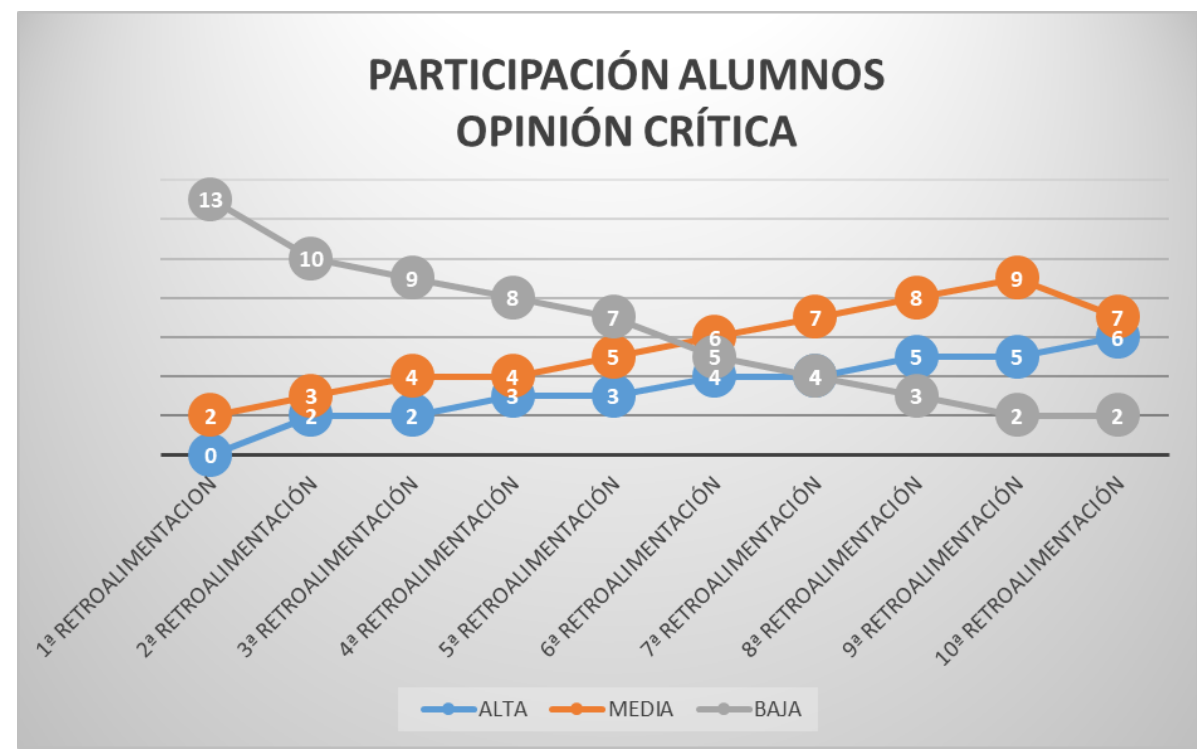

Figura 9. Nivel de participación de los alumnos en las sesiones de retroalimentación.

Fuente: Elaboración propia.

\section{RESULTADOS ENCUESTA FINAL DE SEMESTRE}

En la encuesta que se realiza al final del semestre, se mencionan puntos similares a los de la primera, para poder analizar si hay algún cambio en la opinión de los alumnos respecto al tema de la retroalimentación.

Los resultados fueron altamente positivos referente a ésta, ya que casi un $100 \%$ de los alumnos encuestados respondieron que la retroalimentación les ayudó a su aprendizaje y a la mejora de la calidad de sus proyectos. Mencionando que la retroalimentación les permite conocer el punto de vista de otras personas sobre su proyecto, tanto del docente como del alumno, lo cual les ayudó a encontrar áreas de oportunidad que no podían ver por ellos mismos, logrando así mejores soluciones para las problemáticas de identidad que se les presentaban en los proyectos.

3. Beneficios que la retroalimentación aporta al aprendizaje del alumno en la clase de identidad de la licenciatura en Diseño Gráfico

Con base en los resultados, podemos decir que la retroalimentación ofrece grandes beneficios al aprendizaje del alumno en la clase de identidad de la Licenciatura en Diseño Gráfico, pues pudimos comprobar que hubo un avance significativo en la calidad de los proyectos de los alumnos conforme se realizaron mayor número de sesiones.

Así también podemos observar otro beneficio en cuanto a que favoreció a un mayor desarrollo del espíritu crítico en los alumnos, puesto que conforme se avanzó en el número de sesiones, el nivel de participación en la retroalimentación alumno-alumno aumentó significativamente. 


\section{Propuesta de enseñanza de las Identidades en la Licenciatura en Diseño Gráfico}

En la actualidad, se está trabajando en la propuesta de enseñanza de las Identidades en la Licenciatura en Diseño Gráfico y posteriormente se dará a conocer, para que pueda ser aplicada por los docentes en sus aulas, si así lo consideraran conveniente.

\section{CONCLUSIONES}

La retroalimentación es información que el docente debe aportar al alumno, al respecto de su avance y del aprendizaje mostrado, es fundamental como estrategia de comunicación en el proceso de enseñanza-aprendizaje para llegar a los fines propuestos en una clase.

La retroalimentación alumno-alumno es importante también para el aprendizaje, pero además para el desarrollo del espíritu crítico del alumno con respecto a los conceptos aplicados en la práctica y la calidad de esa aplicación.

Los alumnos, antes de las sesiones, en su mayoría perciben de manera positiva la retroalimentación para su aprendizaje y la mejora de la calidad de sus proyectos.

\section{En la investigación realizada,}

\section{SE OBTUVO UN AVANCE SIGNIFICATIVO}

EN OUANTO ALAMEORA DE LACALIDAD DE LOSPHOYECTOS DE LOS ALMNOS,

conforme fue aumentando el número de sesiones de retroalimentación.

En la investigación realizada, se obtuvo un avance significativo en cuanto a la mejora de la calidad de los proyectos de los alumnos, conforme fue aumentando el número de sesiones de retroalimentación.

Del mismo modo, se observó un aumento en el nivel de participación de los alumnos en cuanto al ejercicio de retroalimentación alumno-alumno, conforme iba avanzando el número de sesiones.

Al final del semestre se muestra una gran satisfacción de los alumnos en cuanto a su aprendizaje, con respecto a la estrategia llevada a cabo basada en la retroalimentación.

En general, podemos concluir que la retroalimentación es importante para el aprendizaje del desarrollo de las Identidades, así también para el desarrollo del espíritu crítico de los alumnos, por tanto es una estrategia que debería estar presente en la enseñanza de esta rama del diseño, para lograr resultados positivos en el aula y un mejor desempeño del alumno en esta área a nivel profesional.

\section{FUENTES DE CONSULTA}

Beltrán, J. (2005), Psicología de la Educación, Editorial Boixareu Universitaria Marcombo, Barcelona. 
García, E. (2015), "La evaluación del aprendizaje: de la retroalimentación a la autorregulación. El papel de las tecnologías", Revista RELIEVE, vol. 21, núm. 2, pp.1-24. ISSN: 1134-4032. [En línea]. Disponible en http:// dx.doi.org/10.7203/relieve.21.2.7546, consultado el 5 de septiembre de 2018.

Gorriez, G. (2017), La importancia de la retroalimentación dentro de la Evaluación Formativa en el Proyecto de Graduación. Escrito en la Facultad No.134, Diseño y Comunicación, Universidad de Palermo. Jornada Académica, Edición 1, Octubre 2017. ISSN 1669-2306. [En línea]. Disponible en http://fido.palermo.edu/servicios_dyc/publicacionesdc/ archivos/675_libro.pdf, consultado el 18 de septiembre de 2018.

Lara, A. (2011), "Agente Adaptable, Aprendizaje y Estructura del Ambiente: Un Enfoque Alternativo", Revista de Economía Institucional, vol. 14, núm. 26, pp. 95-120. ISSN 0124-5996. [En línea]. Disponible en: https:// www.economiainstitucional.com/pdf/No26/alara.pdf, consultado el 5 de septiembre de 2018.

Mendivil, C., Martínez, C. y Camacho, C. (2017), Enseñanza del Diseño Gráfico: Lo que se dice y lo que se hace. I Congreso de Ciencia Sociedad e Investigación Universitaria. Pontifica Universidad Católica del Ecuador. ISSN 2550-679X. [En línea]. Disponible en: http:// repositorio.pucesa.edu.ec/handle/123456789/2061, consultado el 30 de agosto de 2018.

Morales, M. y Restrepo, I. (2015), "Hacer visible el pensamiento: alternativa para una evaluación para el aprendizaje", Revista Infancias Imágenes, vol. 14, núm. 2, pp. 89-100. [En línea]. Disponible en: https://revistas.udistrital.edu.co/ojs/index.php/ infancias/article/view/9075/10884, consultado el 6 de septiembre de 2018.

Rodríguez, J. y Gil, J. (2011), "Las autoevaluaciones y las rúbricas como instrumentos reguladores del aprendizaje. EVALtrends Evaluar para aprender en la universidad". En Experiencias innovadoras en la sistematización de la evaluación, Madrid: Bubok Publishing. pp. 131-145. [En línea]. Disponible en: https://idus.us.es/xmlui/bitstream/ handle/11441/69008/ACTAS2-132-146.pdf? sequence $=1$ \&isAllowed $=y$, consultado el 10 de septiembre de 2018 .

Román, C. (2009), "Sobre la retroalimentación o el feedback en la educación superior on line". Revista Virtual Universidad Católica del Norte, (26), undefined-undefined. ISSN: 0124-5821. [En línea]. Disponible en https://www.redalyc.org/articulo.oa? id=1942/194215516009, consultado el 15 de septiembre de 2018.

Torres, J., Inés, D. y Valdiviezo, P. (2013), "Integración de redes sociales y entornos virtuales de aprendizaje", RED, Revista de Educación a Distancia, núm. 35. ISSN 1578-7680. Universidad de Murcia, España. [En línea]. Disponible en http://www.um.es/ead/red/35/ torres_et_al.pdf, consultado el 5 de septiembre de 2018.

Zatarain, C. (2015), "La didáctica en el diseño gráfico ¿Qué, cómo y para qué enseñar?”, Agentes dinámicos en la enseñanza del diseño gráfico, vol. 1, núm. 1, pp. 9-15. 
